\title{
Exploratory Controlled Study of the Migraine-Suppressing Effects of Psilocybin
}

\author{
Emmanuelle A. D. Schindler ${ }^{1,2,3}$ - R. Andrew Sewell ${ }^{4,5}$ - Christopher H. Gottschalk ${ }^{3}$. Christina Luddy ${ }^{4,5}$. \\ L. Taylor Flynn ${ }^{4,5} \cdot$ Hayley Lindsey ${ }^{1,2,3} \cdot$ Brian P. Pittman ${ }^{4} \cdot$ Nicholas V. Cozzi $^{6,7} \cdot$ Deepak C. D'Souza ${ }^{4,5}$ \\ Accepted: 27 October 2020 / Published online: 12 November 2020 \\ (C) Copyright line is: This is a U.S. government work and not under copyright protection in the U.S.; foreign copyright protection may apply. 2020
}

\begin{abstract}
While anecdotal evidence suggests that select 5-hydroxytryptamine $2 \mathrm{~A}\left(5-\mathrm{HT}_{2 \mathrm{~A}}\right)$ receptor ligands, including psilocybin, may have long-lasting therapeutic effects after limited dosing in headache disorders, controlled investigations are lacking. In an exploratory double-blind, placebo-controlled, cross-over study, adults with migraine received oral placebo and psilocybin $(0.143 \mathrm{mg} / \mathrm{kg})$ in 2 test sessions spaced 2 weeks apart. Subjects maintained headache diaries starting 2 weeks before the first session until 2 weeks after the second session. Physiological and psychological drug effects were monitored during sessions and several follow-up contacts with subjects were carried out to assure safety of study procedures. Ten subjects were included in the final analysis. Over the 2-week period measured after single administration, the reduction in weekly migraine days from baseline was significantly greater after psilocybin (mean, $-1.65(95 \% \mathrm{CI}:-2.53$ to -0.77$)$ days/week) than after placebo $(-0.15(-1.13$ to 0.83 ) days/week; $p=0.003, t(9)=4.11)$. Changes in migraine frequency in the 2 weeks after psilocybin were not correlated with the intensity of acute psychotropic effects during drug administration. Psilocybin was well-tolerated; there were no unexpected or serious adverse events or withdrawals due to adverse events. This exploratory study suggests there is an enduring therapeutic effect in migraine headache after a single administration of psilocybin. The separation of acute psychotropic effects and lasting therapeutic effects is an important finding, urging further investigation into the mechanism underlying the clinical effects of select $5-\mathrm{HT}_{2 \mathrm{~A}}$ receptor compounds in migraine, as well as other neuropsychiatric conditions. Clinicaltrials.gov: NCT03341689
\end{abstract}

Key Words Migraine $\cdot$ headache $\cdot$ preventive treatment $\cdot$ psilocybin $\cdot$ psychedelics

R. Andrew Sewell, posthumous (RAS passed away in 2013)

Emmanuelle A. D. Schindler emmanuelle.schindler@yale.edu

1 Department of Neurology, Yale School of Medicine, New Haven, CT, USA

2 Neurology Service, Veterans Affairs Connecticut Healthcare System, MS 127, 950 Campbell Avenue, West Haven, CT 06516, USA

3 Veterans Affairs Headache Center of Excellence, West Haven, CT, USA

4 Department of Psychiatry, Yale School of Medicine, New Haven, CT, USA

5 Psychiatry Service, Veterans Affairs Connecticut Healthcare System, West Haven, CT, USA

6 Neuropharmacology Laboratory, University of Wisconsin School of Medicine and Public Health, Madison, WI, USA

7 Alexander Shulgin Research Institute, Lafayette, CA, USA

\section{Introduction}

Migraine is one of the most common headache disorders with a prevalence of approximately $15 \%$ and is among the top three disabling diseases worldwide [1-3]. A range of treatment options for migraine exists, though limited efficacy and unpleasant side effects may preclude long-term success [4]. Evidence suggesting that select 5 -hydroxytryptamine $2 \mathrm{~A}\left(5-\mathrm{HT}_{2 \mathrm{~A}}\right)$ receptor agonists, such as psilocybin and lysergic acid diethylamide (LSD), have clinical effects in migraine has existed for over half a century [5-7]. While chemically and pharmacologically similar to other migraine medications (e.g., dihydroergotamine (DHE), methysergide), these particular 5$\mathrm{HT}_{2 \mathrm{~A}}$ agonists are reported to produce long-lasting reductions in headache burden after a single or few oral doses [6, 8-10]. Such a clinical effect is novel and intriguing, though definitive studies are lacking. In the setting of numerous controlled studies with select $5-\mathrm{HT}_{2 \mathrm{~A}}$ agonists in mental health disorders and 
addiction also suggesting long-lasting therapeutic effects after limited dosing [11-17], the demonstration of such effects in headache disorders would suggest that this unique benefit of the drug class seen among different diseases is effected through a shared neurobiological mechanism(s).

The goal of this exploratory, proof-of-concept study was to investigate the effects of psilocybin in migraine in a doubleblind, placebo-controlled, cross-over design. We hypothesized that a single administration of low-dose oral psilocybin in migraine patients would suppress migraine over a 2-week period and be safe in the controlled experimental setting. We were also prepared for unanticipated findings and sought to use all information learned in the design and development of future studies.

\section{Methods}

\section{Regulatory Approvals}

This exploratory study was registered on clinicaltrials.gov (NCT03341689) and received approvals from the Human Studies Subcommittee of Veterans Affairs Connecticut Healthcare System (VACHS) and the Human Investigations Committee of Yale University. The study was conducted under an approved Investigational New Drug application (\#124,874) with the US Food and Drug Administration with Drug Enforcement Administration (DEA) Schedule 1 registration (author DCD).

\section{Psilocybin}

Synthetic psilocybin was prepared under DEA Schedule 1 registration at the University of Wisconsin-Madison (author NVC). Psilocybin was synthesized as described by Shirota et al. [18] with some slight modifications. Chemical analysis to confirm identity and purity included ${ }^{1} \mathrm{H}$ and ${ }^{13} \mathrm{C}$ NMR, HPLC, thermogravimetric analysis, differential scanning calorimetry, and GC-MS. The material was deemed $100 \%$ pure by HPLC. Weight-based capsules of psilocybin $(0.143 \mathrm{mg} / \mathrm{kg})$ and matching placebo (microcrystalline cellulose, obtained from Fagron, St. Paul, MN) were compounded for each subject into identical blue gelatin capsules by the VACHS Investigational Research Pharmacy.

\section{Subjects and Selection Criteria}

Adults (age 21 to 65 years, inclusive), free from serious medical or psychiatric disease, with migraine as defined by the International Classification of Headache Disorders III-beta [19] and with a frequency of migraine attacks of 2 per week or more were eligible to participate in this study. Among the excluded medical conditions were uncontrolled hypertension, coronary artery disease, cardiac arrhythmia, cerebrovascular disease, and serious central or peripheral nervous system or spinal disease (e.g., multiple sclerosis, amyotrophic lateral sclerosis). Psychotic or manic disorders in the subject or a first-degree relative were also exclusionary, as were substance abuse within the past 3 months and any prior serious adverse event with psilocybin, LSD, or related compounds (e.g., mescaline). Prior exposure to psilocybin or related compounds through recreational or medicinal use or through participation in other research studies was not excluded, although any use in the past 3 months was prohibited. Alcohol consumption within 1 week of the first experimental test day was prohibited. Caffeine and nicotine were not restricted. Subjects were required to be free from serotonergic antidepressants (e.g., fluoxetine) for at least 6 weeks, serotonergic antiemetics (e.g., ondansetron) for at least 2 weeks, and vasoconstrictive medications (e.g., pseudoephedrine) for at least five elimination half-lives of said medication. Triptans (e.g., sumatriptan) were permitted, but no more than twice weekly and not within five elimination half-lives of said triptan before each test day nor within five elimination half-lives $(15 \mathrm{~h})$ of psilocin, the active metabolite of psilocybin, after each test day.

\section{Recruitment and Screening}

Subjects were recruited from the local community, headache centers, online headache websites, and word of mouth. Interested candidates were informed of the study and prescreened over the telephone. If candidates passed the prescreen based on the study criteria, they were invited for a full evaluation to assess eligibility. This included a medical history, physical examination, laboratory tests (hematology, chemistry, liver and thyroid studies, urinalysis, urine toxicology, urine pregnancy, electrocardiogram), structured mental health interview, personality assessment, and verbal intelligence quotient test. In addition to research staff taking detailed histories to verify the diagnosis of migraine and other medical and psychiatric conditions, subjects' physicians were contacted in order to corroborate their migraine diagnosis and inquire about medical, psychiatric, and substance use history; written consent for this physician contact was required for study participation. Written consent was also obtained to speak with a family member or friend in order to exclude any additional safety concerns for study participation. During the multistage screening process, study procedures and the physiological and psychological effects of psilocybin were repeatedly reviewed with subjects. Subjects were also quizzed on study procedures, the expected effects of psilocybin, and emergency contacts. In compliance with the Helsinki Declaration of 1975, as revised in 2000 [20], informed consent was obtained from every subject who participated in the study. Furthermore, subjects were informed that they could 
decline to participate in the study without penalty and were free to withdraw from the study at any time. The sources of study funding were also disclosed to all participants.

\section{Study Design}

This was an exploratory double-blind, placebo-controlled, crossover study. Subjects completed 2 experimental sessions, separated by at least 14 days, under a standard approved blinding procedure, in which drug dose and order of administration were unknown to subjects and research staff. The blinded procedure was also reflected in the clinicaltrials.gov registration. In the first experimental session, all subjects received an oral placebo capsule, and in the second experimental session, all subjects received an identically appearing oral psilocybin capsule. In this design, each subject acted as his own control and placebo was given first so that the potential long-term effects of psilocybin, if given first, would not interfere with placebo treatment, if given second.

\section{Assessment of Migraine Burden}

Subjects maintained a headache diary starting 2 weeks before the first experimental session until 2 weeks after the second experimental session. Subjects were required to document every headache attack, migraine (with associated migrainous symptoms), or otherwise (without associated migrainous symptoms; not counted as a migraine attack in the analysis). Migraine attack pain and associated symptoms-photophobia (light sensitivity), phonophobia (sound sensitivity), nausea/vomiting - as well as attack-related functional impairment were documented using a 0-3 numerical scale $(0=$ none, $1=$ mild, $2=$ moderate, $3=$ severe). Abortive medications taken and their effects were also recorded in the diary. Only the 14 days prior to the first experimental session (baseline) and the 14 days after each experimental session (drug effect) were counted in the final analysis.

\section{Experimental Sessions}

At 8:00 am, subjects reported to the Neurobiological Studies Unit (NSU), an outpatient research facility and the location of neuropsychopharmacologic studies with investigational drugs at $\mathrm{VACHS} /$ Yale for $>25$ years. Urine drug, urine pregnancy (when applicable), and alcohol breathalyzer tests were done and required to be negative in order to proceed. After a light breakfast, an intravenous line was placed (for potential rescue medication) and baseline measures were collected (see below). Subjects typically ingested the drug capsule between 8:30 am and 9:30 am. Blood pressure, heart rate, and peripheral oxygenation were measured at baseline, every $15 \mathrm{~min}$ for the first hour, every $30 \mathrm{~min}$ for the second hour, and then every hour thereafter. General drug effects ("overall," "anxiety/fear," "sleepiness/sedation," "nausea," "joy/intense happiness," "peace/harmony") were self-reported on a $0-3$ visual analog scale (VAS; $0=$ none, $1=$ minimal, $2=$ moderate, $3=$ definite) at baseline, every $30 \mathrm{~min}$ for the first $2 \mathrm{~h}$, and then hourly thereafter. The rating of "overall" drug effect served as a means for subjects to report the integrated sensation that they had received a drug. Psychedelic effects were self-reported at the end of experimental sessions using the validated 5Dimensional Altered States of Consciousness (5D-ASC) scale, which is divided into the following subscales: oceanic boundlessness (OBN), dread of ego dissolution (DED), visionary restructuralization (VRS), acoustic alterations (AUA), and vigilance reduction (VIR) [21]. Subjects were discharged from the NSU no sooner than $6 \mathrm{~h}$ after capsule ingestion and not until physiological and psychological drug effects had resolved. Subjects were not allowed to drive themselves after experimental sessions. Emergency contacts, including 24-h/7-day psychiatry services, were provided to all subjects.

\section{Follow-up and Payment}

Telephone follow-up was performed by a research team member familiar to the subject the day after and weekly for 2 weeks after each experimental session and then at approximately 2 and 3 months. Subjects were asked about adverse events (AEs), physical health, and psychological health, and any questions they had were answered. After all subjects completed study procedures, subjects were contacted by telephone and told what they had received during experimental sessions. Subjects were paid US $\$ 100$ per experimental session and US $\$ 50$ for in-person screening.

\section{Outcome Measures}

The primary outcome measure was the change in migraine frequency, measured as the change in weekly migraine days compared to baseline in the 2 weeks after drug administration. Other primary outcome measures included change in weekly migraine attacks, light sensitivity, sound sensitivity, nausea/ vomiting, and attack-related functional impairment in the 2 weeks after drug administration. Secondary outcome measures included change in the use of migraine abortives, time to the next migraine attack, acute changes in vital signs, general drug effects, psychotropic ratings, and adverse events. As this study was exploratory, minor changes to outcomes, which did not alter findings or conclusions, were made and are detailed in the Supplemental Methods.

\section{Statistical Analysis}

A total of 12 subjects was sought for this exploratory study. For within-subject analyses with a two-tailed $\alpha=0.05,12$ subjects would provide $80 \%$ statistical power to detect large effects $\left(d^{\prime}=0.9\right.$ ). Our final sample of $n=10$ (Fig. 1) provided $80 \%$ statistical power to detect large effects $\left(d^{\prime}=1.0\right)$. 
Fig. 1 Subject screening and enrollment

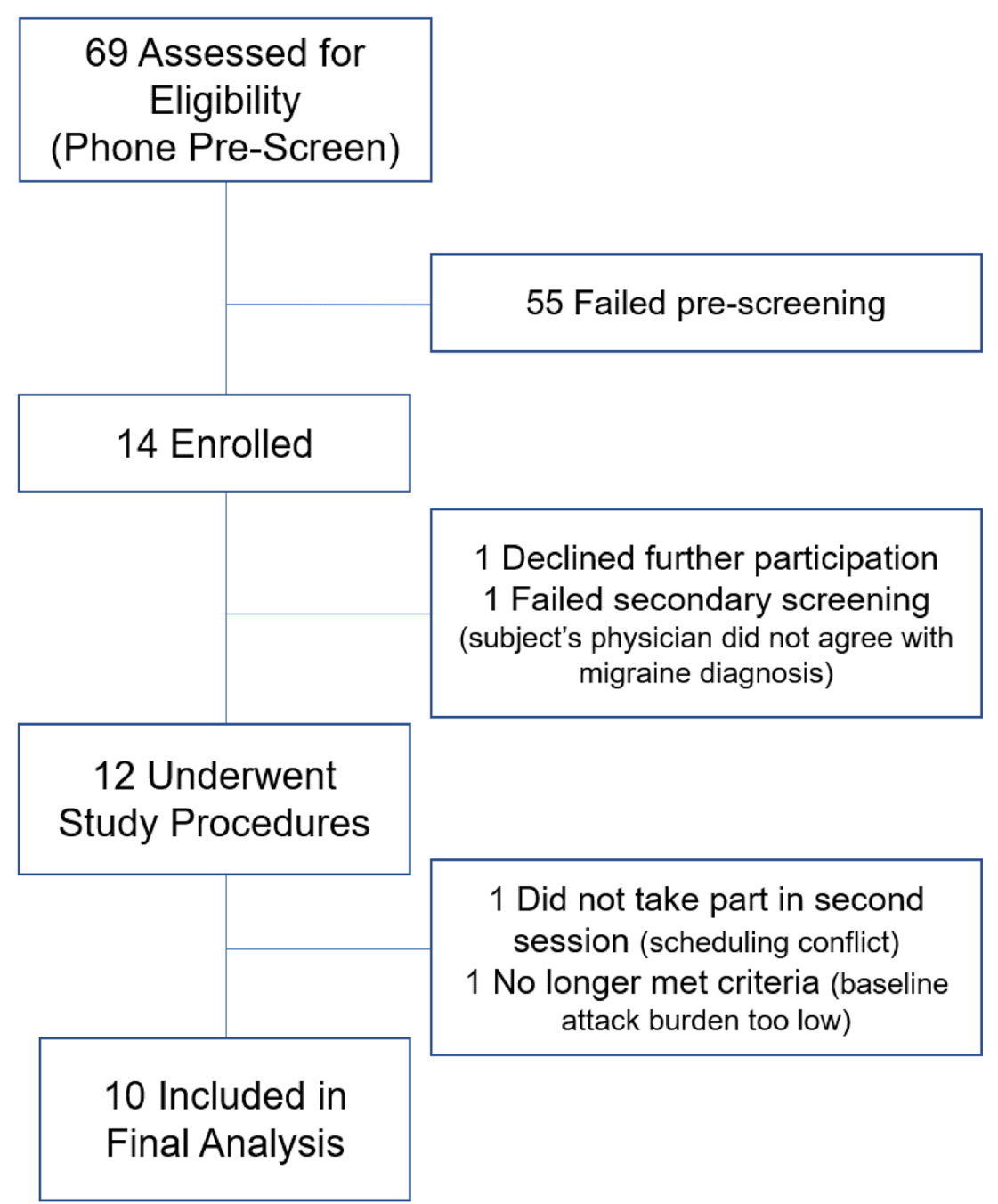

Statistical analyses were performed using SAS, version 9.4 (SAS Institute Inc., Cary, NC) and figures were produced with GraphPad Prism (GraphPad Software Inc., La Jolla, CA). All statistical tests were two-sided with an overall prehypothesis alpha threshold of 0.05 . Measures of error are shown as standard error about the mean (SEM) or $95 \%$ confidence interval (95\% CI). The changes from baseline in migraine burden as measured in the headache diary were calculated as raw values and compared between placebo and psilocybin via paired $t$ test. The analysis plan developed by the statistical consult (author BPP) considered alternatives (including analysis of variance), which did not affect the results. The time to the first and second migraine attacks after drug administration was also compared between placebo and psilocybin via paired $t$ test. When no migraine attacks occurred for this measure, "15 days" was used as the time to that attack. Acute effects of drug administration on mean arterial pressure (MAP), heart rate, peripheral oxygenation, and general drug effects measured throughout the session were analyzed using linear mixed models which included treatment and time as within- subject effects and random subject effects. The best-fitting variance-covariance structure was based on information criteria. Least square means were compared post hoc to determine the nature of significant interactions. Psychotropic effects as measured by the 5D-ASC scale were calculated as a percent of the total possible score $[14,22]$ and compared using a linear mixed model with treatment and dimension (see scale dimensions) included as within-subject factors and random subject effects. Potential associations between general drug or psychotropic effects and the change in weekly migraine days were assessed using correlation (Spearman) analysis. The numbers of AEs were compared between placebo and psilocybin using Fisher's exact test.

\section{Results}

Between November 2017 and December 2019, patients were assessed for study eligibility. A total of 69 candidates was prescreened; 14 underwent secondary screening and 12 
underwent study procedures. Ten subjects were included in the final analysis (Fig. 1). Two subjects were excluded from final analysis; 1 was unable to participate in the second experimental session for scheduling conflicts and 1 subject's baseline period did not contain enough migraine attacks for qualification.

\section{Demographics, Migraine Characteristics, and Substance Use (Table 1)}

Seven females and 3 males were included in the final analysis. The average age was 40.5 (SEM 4.4) years. Two subjects had previously tried psilocybin (not specifically for migraine treatment). At the time of enrollment, only 1 subject indicated that they were satisfied with their current migraine treatment regimen and all subjects indicated that they would at least consider trying a new migraine treatment if it were available.

\section{Migraine Burden in the 2 Weeks After Drug Administration}

Migraine Frequency The change from baseline in weekly migraine days showed a significantly greater reduction after psilocybin (mean, -1.65 (95\% CI: -2.53 to -0.77$)$ ) than after placebo $(-0.15$ ( -1.13 to 0.83$) ; p=0.003, t(9)=4.11$; Fig. 2 , Table 2). The percentages of subjects who had at least $25 \%$, $50 \%$, and $75 \%$ reductions in weekly migraine days were as follows: $80 \%, 50 \%, 30 \%$ after psilocybin, and 20\%, 20\%, 0\% after placebo, respectively. Psilocybin and placebo significantly differed at the level of at least $25 \%$ reduction $(p=$ 0.023; Fisher exact).

Other Migraine Outcomes Reductions from baseline were significantly greater after psilocybin compared to placebo in weekly migraine attacks, pain severity, attack-related functional impairment, and weekly migraine abortive days (Table 2). There were no significant differences on migraine attack duration or associated symptom (photophobia, phonophobia, nausea/vomiting) ratings.

Time to Next Migraine Attacks Given that psilocybin is known to acutely induce headache attacks (see AE) [23], the times to both the first and second migraine attack were measured. The time to the first attack was statistically equivocal, but the time to the second attack was significantly greater after psilocybin (10.30 (1.61) days) than after placebo (5.00 (1.13) days; $p=$ $0.012, t(9)=3.14$; Fig. 3 ).

\section{Acute Effects of Drug Administration}

General Drug Effects During experimental sessions, an interaction between drug and time was observed for "overall drug effects" $(F(8,142)=3.74, p=0.0005)$, where psilocybin $(F(8$,
$142)=11.3, p<0.0001)$ but not placebo $(F(8,142)=0.57$, $p=0.80$ ) elicited "overall drug effects" (Suppl Table). A significant interaction was also observed for the feeling of "peace/harmony," where psilocybin, but not placebo, elicited this feeling. No interactions were observed for other general drug effects. It is noted that only 1 subject remained in the NSU for an additional $60 \mathrm{~min}$ following the requisite $6 \mathrm{~h}$ after psilocybin administration to allow general drug effects to dissipate. The maximum "overall drug effect" rating during psilocybin exposure did not correlate with the percent change from baseline in weekly migraine days $(r=0.469 ; p=0.17)$.

Psychedelic Effects Subjects scored the 5D-ASC scale at the end of each experimental session. The percent possible score for the total scale was significantly higher after psilocybin $(19.35 \%(7.55))$ as compared to placebo (3.08\% (1.80); $p=$ $0.026, t(9)=2.65$ ). In the mixed model comparing treatment across individual dimensions, the interaction between treatment and dimension was not significant $(F(4,81)=2.22$, $p=0.07)$. The percent total 5D-ASC scale score during psilocybin exposure did not correlate with the percent change from baseline in weekly migraine days $(r=0.418 ; p=0.23)$.

\section{Adverse Events}

There were no serious or unexpected AEs in this study. During experimental sessions, lightheadedness and tension/ sore muscles were reported with both placebo and psilocybin administration (Table 3). In the $24 \mathrm{~h}$ after experimental sessions, both placebo and psilocybin administration were followed by tension/sore muscles, general headache attack, and migraine attack (Table 3). There were no significant differences between placebo and psilocybin in the incidences of AEs, except that there was a significant drug $\times$ time interaction for MAP over the experimental test day $(F(10,186)=2.52$, $p=0.007$; Suppl Table). Post hoc analysis revealed a significant increase in MAP with psilocybin administration starting at $45 \mathrm{~min}$ until $4 \mathrm{~h}$ after ingestion. The maximum acute increase in MAP over placebo was 12.2 (4.61 to 19.73) $\mathrm{mmHg}$ at $1.5 \mathrm{~h}$ after ingestion. All AEs were transient and selflimiting (i.e., no rescue medications required), and no subjects withdrew from the study due to an AE. During follow-up with subjects, there were no AEs warranting professional intervention. At 3 months' follow-up, all subjects denied any lasting physical, psychological, or cognitive changes.

\section{Discussion}

This exploratory, proof-of-concept, double-blind, placebocontrolled, cross-over investigation showed significant reductions in migraine measures in the 2 weeks assessed after the single administration of a low oral dose of psilocybin. To our 
Table 1 Demographics, migraine characteristics, and substance use

\begin{tabular}{|c|c|c|c|c|}
\hline Characteristic/behavior/question & Mean (SEM) & \multicolumn{2}{|l|}{ Range } & \\
\hline Sex & $7 \mathrm{~F}: 3 \mathrm{M}$ & \multicolumn{2}{|l|}{ - } & \\
\hline Age (years) & $40.5(4.4)$ & \multicolumn{2}{|l|}{23 to 63} & \\
\hline Age of migraine onset (years) & $18.7(2.9)$ & \multicolumn{2}{|l|}{6 to 35} & \\
\hline Race & Caucasian $100 \%$ & \multicolumn{2}{|l|}{-} & \\
\hline Weight $(\mathrm{kg})$ & $65.4(4.9)$ & \multicolumn{2}{|l|}{47.7 to 95.9} & \\
\hline \multirow[t]{2}{*}{ Body mass index (BMI; $\mathrm{kg} / \mathrm{m}^{2}$ ) } & $22.9(1.6)$ & \multicolumn{2}{|l|}{18.1 to 32.1} & \\
\hline & & Yes & No & Other \\
\hline \multicolumn{2}{|l|}{ Family history of migraine } & 7 & 1 & 2 (unsure) \\
\hline \multirow[t]{8}{*}{ Migraine attack triggers } & Alcohol & 9 & 1 & \\
\hline & Strong smells & 8 & 2 & \\
\hline & Bright/flashing lights & 6 & 4 & \\
\hline & Weather changes & 10 & 0 & \\
\hline & Altitude changes & 5 & 5 & \\
\hline & Travel across time zones & 1 & 9 & \\
\hline & Other & 8 & 2 & \\
\hline & No triggers & 0 & 10 & \\
\hline $\begin{array}{l}\text { Have you ever experienced a negative side effect } \\
\text { from a medication you took for migraine headache? }\end{array}$ & & 9 & 1 & \\
\hline Are you satisfied with your current medication regimen? & & 1 & 6 & 3 (somewhat) \\
\hline $\begin{array}{l}\text { If there were a new medication available to treat } \\
\text { migraine headache, would you try it? }\end{array}$ & & 8 & 0 & 2 (perhaps) \\
\hline Current alcohol use & & 7 & 3 (quit) & \\
\hline \multirow{6}{*}{$\begin{array}{l}\text { Past use of controlled substances (for any purpose, } \\
\text { excluding alcohol and nicotine) }\end{array}$} & Psilocybin and related & 2 & 8 & \\
\hline & Cannabinoids & 9 & 1 & \\
\hline & Opioids & 6 & 4 & \\
\hline & Stimulants & 6 & 4 & \\
\hline & Other & 5 & 5 & \\
\hline & None & 1 & 9 & \\
\hline Past alcohol/drug abuse/dependence & & 1 (remission) & 9 & \\
\hline
\end{tabular}

"Psilocybin and related" include psilocybin, lysergic acid diethylamide (LSD), $N, N$-dimethyltryptamine (DMT), and mescaline. "Stimulants" include cocaine, 3,4-methylenedioxymethamphetamine (MDMA, ecstasy), and amphetamines. "Other" includes benzodiazepines, barbiturates, and phencyclidine

$\mathrm{SEM}=$ standard error about the mean

knowledge, this is the first controlled study of psilocybin in a headache disorder. The findings from this study validate the previous anecdotal reports of therapeutic effects in migraine and complement research in past decades with psilocybin and other select $5-\mathrm{HT}_{2 \mathrm{~A}}$ receptor agonists demonstrating lasting beneficial effects in treating depression [11, 12, 24], anxiety $[11,13,14]$, alcohol addiction $[16,25,26]$, and cigarette smoking $[15,17]$.

To our knowledge, the therapeutic effect over 2 weeks after the single administration of an oral agent reported in this study is a novel finding in migraine therapy. This contrasts with existing preventive migraine therapies that necessitate repeated, daily administration (e.g., topiramate) or include treatments that remain in the body long after administration (e.g., anti-calcitonin gene-related peptide or receptor monoclonal antibodies). Lasting clinical effects after relatively limited drug administration are seen with such conventional transitional migraine treatments as corticosteroids, which are administered in oral pulses of various duration, and DHE, which is administered as a thrice daily, 5-day intravenous or subcutaneous injection regimen [27]. It is notable that DHE also has agonist activity at the 5- $\mathrm{HT}_{2 \mathrm{~A}}$ receptor, in addition to several other receptors [28]. Whether a shared mechanism of action in migraine exists between psilocybin and DHE or psilocybin and corticosteroids will require further study.

In contrast to some previous psilocybin studies for other neuropsychiatric conditions $[11,17]$, the current study did not find that psychotropic effects correlated with the migraine 


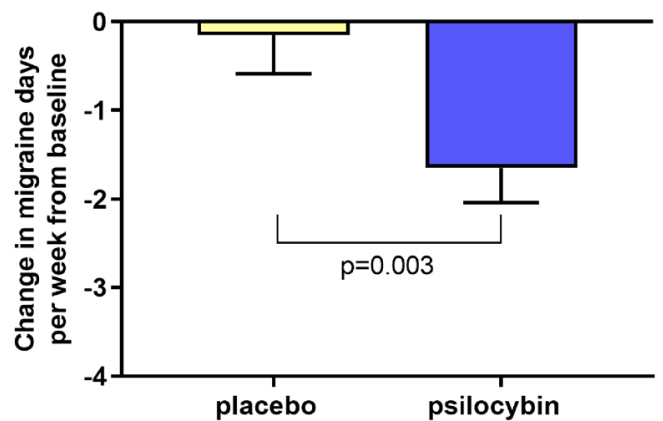

Fig. 2 Change in migraine frequency. The average reduction in weekly migraine days from baseline in the 2 weeks following the single administration of drug was significantly greater after psilocybin than after placebo ( $n=10$, paired $t$ test). Error bars represent standard error about the mean

frequency change over 2 weeks, suggesting that the therapeutic effect of psilocybin in migraine is independent of acute changes in sensation and perception. In fact, subjects in the present study with the highest 5D-ASC scale scores had some of the smallest reductions in migraine burden, fitting with the positive coefficient calculated. These observations are consistent with survey studies reporting that subhallucinogenic doses of psilocybin and LSD provide prophylactic relief in headache disorders $[6,8,9]$. Furthermore, a congener of LSD, 2-bromo-lysergic acid diethylamide (BOL-148 or $\mathrm{BOL}$ ), which has greatly reduced psychotropic effects, is also reported to have medicinal effects in cluster and other headache disorders [7, 8, 29]. Collectively, these findings suggest dissociation between the acute psychotropic effects and the sustained therapeutic action of psilocybin and other select 5$\mathrm{HT}_{2 \mathrm{~A}}$ receptor compounds in headache disorders. If confirmed, this raises the intriguing possibility that the therapeutic effects of these particular compounds may not require their namesake "psychedelic" effects.

While encouraged by the findings in this exploratory study, before this approach could be used clinically, it is imperative that additional controlled investigations be completed in order to understand psilocybin's full capacity to suppress migraine, as well as its long-term safety and tolerability. To verify the present findings, it will be necessary to replicate the results of this study in a larger sample under a fully randomized design. Studies with a dose range will inform on whether the effects of psilocybin in migraine are dose dependent. Studies investigating repeated administration either in close succession or separated by specified intervals will help illustrate psilocybin's abilities as a transitional and/or preventive treatment. In parallel with clinical investigations, studies determining the mechanisms of psilocybin's effects will inform on the biological target of this agent and potential for drug development, including chemical modifications that can minimize unnecessary effects while maximizing reductions in migraine parameters. For instance, it has been proposed that the immunomodulatory and anti-inflammatory effects common to these select

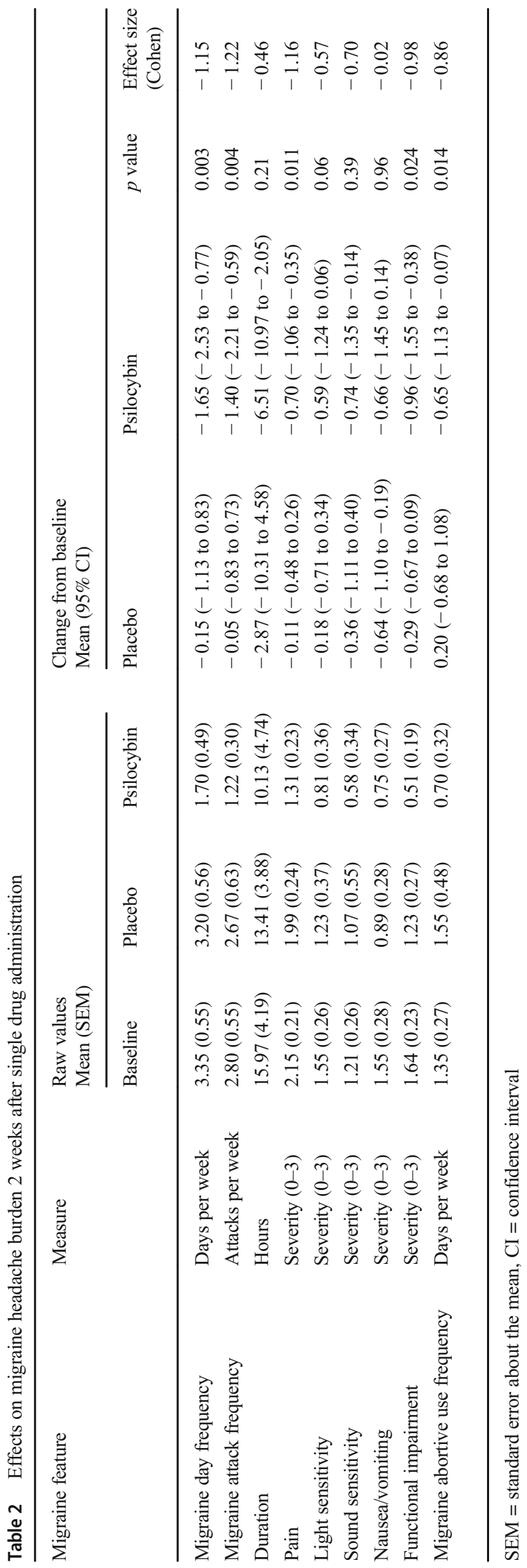




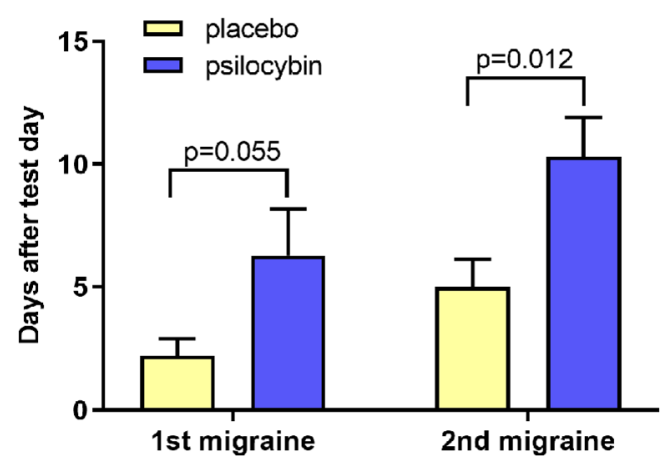

Fig. 3 Time to the next migraine attacks. The average number of days to the first and second migraine attacks after drug administration is shown. The time to the second attack was significantly greater after psilocybin than after placebo ( $n=10$, paired $t$ test). Error bars represent standard error about the mean

5- $\mathrm{HT}_{2 \mathrm{~A}}$ receptor compounds involve interactions among serotonin, sigma-1, and toll-like receptors [30], all of which are implicated in migraine, itself a chronic inflammatory condition [31-34]. Neuroendocrine systems and sleep also underlie both migraine pathophysiology and the known actions of 5$\mathrm{HT}_{2 \mathrm{~A}}$ receptor compounds [35-37].

This study has several strengths and limitations. The double-blind, placebo-controlled, cross-over design is a powerful approach. The sample size is small, though appropriate for an exploratory, proof-of-concept investigation. Strong statistical significance and large effect sizes validate the findings in this small sample. All subjects were Caucasian and had relatively high starting headache burden, and while not representative of the general migraine population, it nevertheless allowed for exploring the effects and safety in a proof-of-concept study. We also included subjects meeting criteria for either episodic or chronic migraine in the present study. Subgroup analysis did not reveal significant differences between chronic and episodic subjects ( $n=5$ each; data not shown). There were also no obvious age or sex differences (data not shown), though future, adequately powered studies will be necessary to conclusively determine whether any differences between subgroups exist. The outcome measure of time to next migraine was confounded by ceiling effects, as 2 subjects had only 1 migraine attack and 2 had no migraine attacks in the 2 weeks after psilocybin administration. A longer duration of the headache diary in future studies would help better characterize the headache-free period after drug administration. Validated screens of migraine severity were not employed in this study, though such tools as the Global Assessment of Migraine Severity (GAMS), Migraine Disability Assessment (MIDAS), or Headache Impact Test-6 (HIT-6) could capture this measure, particularly in longer-term studies. Lastly, psilocybin did induce some physiological and psychotropic effects, which might suggest to subjects when they received the drug (i.e., unblinding). "Overall drug effect" and psychedelic effects did not correlate with migraine frequency reduction, however. Furthermore, these same acute drug effects were also reported after placebo administration, indicating some success in the blinding procedure. Future studies using a control agent that has similar acute drug effects to psilocybin, such as niacin, could reduce the risk of unblinding. Quantifiable measures of subject blinding, as well as expectation, were not included in the present study, but should be incorporated into future studies to help determine the impact of these confounds on the results.

In the first controlled investigation of psilocybin in migraine, we have demonstrated migraine-suppressing effects in the 2 weeks measured after the single administration of a low oral dose. The change in migraine frequency

Table 3 Adverse event record

\begin{tabular}{|c|c|c|c|c|c|c|}
\hline \multirow[t]{2}{*}{ Adverse event } & \multicolumn{3}{|c|}{ Developed during test day } & \multicolumn{3}{|c|}{ Developed in the next $24 \mathrm{~h}$} \\
\hline & Placebo & Psilocybin & $\begin{array}{l}p \text { value } \\
\text { (Fisher } \\
\text { exact) }\end{array}$ & Placebo & Psilocybin & $\begin{array}{l}p \text { value } \\
\text { (Fisher } \\
\text { exact) }\end{array}$ \\
\hline Lightheadedness & 1 & 3 & 0.58 & 0 & 0 & 1.0 \\
\hline Nausea & 0 & 4 & 0.09 & 0 & 0 & 1.0 \\
\hline Anxiety & 0 & 3 & 0.21 & 0 & 0 & 1.0 \\
\hline Tingling/paresthesia & 0 & 1 & 0.99 & 0 & 0 & 1.0 \\
\hline Cold/shivering & 0 & 1 & 0.99 & 0 & 0 & 1.0 \\
\hline Dry mouth & 0 & 1 & 0.99 & 0 & 0 & 1.0 \\
\hline Confusion & 1 & 0 & 0.99 & 0 & 0 & 1.0 \\
\hline Tension/sore muscles & 3 & 1 & 0.58 & 2 & 2 & 1.0 \\
\hline Headache attack (general) & 0 & 0 & 1.0 & 2 & 5 & 0.35 \\
\hline Migraine attack & 0 & 1 & 0.99 & 2 & 2 & 1.0 \\
\hline IV site induration & 0 & 0 & 1.0 & 0 & 1 & 0.99 \\
\hline
\end{tabular}

IV = intravenous line 
was independent from acute psychotropic effects. This exploratory study supports the viability of psilocybin as an investigational agent in migraine and shows that with careful recruitment, screening, preparatory, monitoring, and follow-up procedures [38], low-dose psilocybin can safely be administered orally to migraine patients in the experimental research setting. This study also represents a new arm in the field of select $5-\mathrm{HT}_{2 \mathrm{~A}}$ receptor compounds, offering a new perspective on the unique abilities of this drug class.

Supplementary Information The online version contains supplementary material available at https://doi.org/10.1007/s13311-020-00962-y.

Acknowledgments We thank CH-TAC, LLC, and the Schizophrenia Neuropharmacology Research Group at Yale (SNRGY) for their support of this project. This study was conducted with assistance from VACHS. Any opinions, findings, and conclusions or recommendations expressed in this material are those of the authors and do not necessarily reflect the views of the Department of Veterans Affairs. The nurses of the NSU where this study was conducted, Ms. Angelina Genovese, Elizabeth O'Donnell, and Margaret Dion-Marovitz, as well as Rachel Galvan, PharmD, of the VACHS Investigational Research Pharmacy, are thanked for their excellent support. A vital force in the foundation of this research was the late Dr. Sewell, who was tragically lost to us in 2013 and whom we honor with the publication of this work

Required Author Forms Disclosure forms provided by the authors are available with the online version of this article.

\section{Compliance with Ethical Standards}

This exploratory study was registered on clinicaltrials.gov (NCT03341689) and received approvals from the Human Studies Subcommittee of Veterans Affairs Connecticut Healthcare System (VACHS) and the Human Investigations Committee of Yale University. In compliance with the Helsinki Declaration of 1975, as revised in 2000 [20], informed consent was obtained from every subject who participated in the study.

\section{References}

1. Lipton RB, Stewart WF, Diamond S, Diamond ML, Reed M. Prevalence and burden of migraine in the United States: data from the American Migraine Study II. Headache. 2001;41(7):646-657.

2. Burch R, Rizzoli P, Loder E. The Prevalence and Impact of Migraine and Severe Headache in the United States: Figures and Trends From Government Health Studies. Headache. 2018;58(4): 496-505.

3. GBD Disease and Injury Incidence and Prevalence Collaborators. Global, regional, and national incidence, prevalence, and years lived with disability for 328 diseases and injuries for 195 countries, 1990-2016: a systematic analysis for the Global Burden of Disease Study 2016. Lancet 2017;390(10100):1211-1259.

4. Hepp Z, Dodick DW, Varon SF, Gillard P, Hansen RN, Devine EB. Adherence to oral migraine-preventive medications among patients with chronic migraine. Cephalalgia 2015;35(6):478-488.

5. Sewell RA, Gottschalk CH. Problem child is no headache. Headache. 2011;51(2):306; author reply -7.
6. Andersson M, Persson M, Kjellgren A. Psychoactive substances as a last resort-a qualitative study of self-treatment of migraine and cluster headaches. Harm Reduct J 2017;14(1):60.

7. Sicuteri F. Prophylactic Treatment of Migraine by Means of Lysergic Acid Derivatives. Triangle 1963;6:116-125.

8. Schindler EA, Gottschalk CH, Weil MJ, Shapiro RE, Wright DA, Sewell RA. Indoleamine Hallucinogens in Cluster Headache: Results of the Clusterbusters Medication Use Survey. J Psychoactive Drugs 2015;47(5):372-381.

9. Sewell RA, Halpern JH, Pope HG, Jr. Response of cluster headache to psilocybin and LSD. Neurology. 2006;66(12):1920-1922.

10. Johnson S, Black QC. Can Psychedelics Alleviate Symptoms of Cluster Headache and Accompanying Mental Health Problems? A Case Report Involving Hawaiian Baby Woodrose. J Psychoactive Drugs 2020;52(4):319-323.

11. Griffiths RR, Johnson MW, Carducci MA, Umbricht A, Richards WA, Richards BD, et al. Psilocybin produces substantial and sustained decreases in depression and anxiety in patients with lifethreatening cancer: A randomized double-blind trial. J Psychopharmacol 2016;30(12):1181-1197.

12. Carhart-Harris RL, Bolstridge M, Day CMJ, Rucker J, Watts R, Erritzoe DE, et al. Psilocybin with psychological support for treatment-resistant depression: six-month follow-up. Psychopharmacology. 2018;235(2):399-408.

13. Gasser P, Kirchner K, Passie T. LSD-assisted psychotherapy for anxiety associated with a life-threatening disease: a qualitative study of acute and sustained subjective effects. J Psychopharmacol 2015;29(1):57-68.

14. Grob CS, Danforth AL, Chopra GS, Hagerty M, McKay CR, Halberstadt AL, et al. Pilot study of psilocybin treatment for anxiety in patients with advanced-stage cancer. Arch Gen Psychiatry 2011;68(1):71-78.

15. Johnson MW, Garcia-Romeu A, Griffiths RR. Long-term followup of psilocybin-facilitated smoking cessation. Am J Drug Alcohol Abuse 2017;43(1):55-60.

16. Bogenschutz MP, Forcehimes AA, Pommy JA, Wilcox CE, Barbosa PC, Strassman RJ. Psilocybin-assisted treatment for alcohol dependence: a proof-of-concept study. J Psychopharmacol 2015;29(3):289-299.

17. Garcia-Romeu A, Griffiths RR, Johnson MW. Psilocybinoccasioned mystical experiences in the treatment of tobacco addiction. Curr Drug Abuse Rev 2014;7(3):157-164.

18. Shirota O, Hakamata W, Goda Y. Concise large-scale synthesis of psilocin and psilocybin, principal hallucinogenic constituents of "Magic Mushroom”. J Nat Prod 2003;66(6):885-887.

19. Headache Classification Committee of the International Headache $\mathrm{S}$. The International Classification of Headache Disorders, 3rd edition (beta version). Cephalalgia 2013;33(9):629-808.

20. World Medical Association Declaration of Helsinki: ethical principles for medical research involving human subjects. JAMA 2000;284(23):3043-3045.

21. Studerus E, Kometer M, Hasler F, Vollenweider FX. Acute, subacute and long-term subjective effects of psilocybin in healthy humans: a pooled analysis of experimental studies. J Psychopharmacol 2011;25(11):1434-1452.

22. Hasler F, Grimberg U, Benz MA, Huber T, Vollenweider FX. Acute psychological and physiological effects of psilocybin in healthy humans: a double-blind, placebo-controlled dose-effect study. Psychopharmacology. 2004;172(2):145-156.

23. Johnson MW, Sewell RA, Griffiths RR. Psilocybin dosedependently causes delayed, transient headaches in healthy volunteers. Drug Alcohol Depend 2012;123(1-3):132-140.

24. Sanches RF, de Lima Osorio F, Dos Santos RG, Macedo LR, Maiade-Oliveira JP, Wichert-Ana L, et al. Antidepressant Effects of a Single Dose of Ayahuasca in Patients With Recurrent Depression: A SPECT Study. J Clin Psychopharmacol 2016;36(1):77-81. 
25. Krebs TS, Johansen PO. Lysergic acid diethylamide (LSD) for alcoholism: meta-analysis of randomized controlled trials. J Psychopharmacol 2012;26(7):994-1002.

26. Bogenschutz MP, Podrebarac SK, Duane JH, Amegadzie SS, Malone TC, Owens LT, et al. Clinical Interpretations of Patient Experience in a Trial of Psilocybin-Assisted Psychotherapy for Alcohol Use Disorder. Front Pharmacol 2018;9:100.

27. Nagy AJ, Gandhi S, Bhola R, Goadsby PJ. Intravenous dihydroergotamine for inpatient management of refractory primary headaches. Neurology. 2011;77(20):1827-1832.

28. Silberstein SD, McCrory DC. Ergotamine and dihydroergotamine: history, pharmacology, and efficacy. Headache. 2003;43(2):144166.

29. Karst M, Halpern JH, Bernateck M, Passie T. The non-hallucinogen 2-bromo-lysergic acid diethylamide as preventative treatment for cluster headache: an open, non-randomized case series. Cephalalgia 2010;30(9):1140-1144.

30. Szabo A. Psychedelics and Immunomodulation: Novel Approaches and Therapeutic Opportunities. Front Immunol 2015;6:358.

31. Anderson TR, Andrew RD. Spreading depression: imaging and blockade in the rat neocortical brain slice. J Neurophysiol 2002;88(5):2713-2725.

32. Kwon YB, Jeong YC, Kwon JK, Son JS, Kim KW. The Antinociceptive Effect of Sigma-1 Receptor Antagonist, BD1047, in a Capsaicin Induced Headache Model in Rats. Korean J Physiol Pharmacol 2009;13(6):425-429.
33. Conti P, D'Ovidio C, Conti C, Gallenga CE, Lauritano D, Caraffa A, et al. Progression in migraine: Role of mast cells and proinflammatory and anti-inflammatory cytokines. Eur J Pharmacol 2019;844:87-94.

34. Goadsby PJ, Wietecha LA, Dennehy EB, Kuca B, Case MG, Aurora SK, et al. Phase 3 randomized, placebo-controlled, double-blind study of lasmiditan for acute treatment of migraine. Brain J Neurol 2019;142(7):1894-1904.

35. Li W, Diao X, Chen C, Li C, Zhang Y, Li Y. Changes in hormones of the hypothalamic-pituitary-gonadal axis in migraine patients. $\mathrm{J}$ Clin Neurosci 2018;50:165-171.

36. Schindler EAD, Wallace RM, Sloshower JA, D'Souza DC. Neuroendocrine Associations Underlying the Persistent Therapeutic Effects of Classic Serotonergic Psychedelics. Front Pharmacol 2018;9:177.

37. Kuypers KPC. Psychedelic medicine: The biology underlying the persisting psychedelic effects. Med Hypotheses 2019;125:21-24.

38. Johnson M, Richards W, Griffiths R. Human hallucinogen research: guidelines for safety. J Psychopharmacol 2008;22(6):603620.

Publisher's Note Springer Nature remains neutral with regard to jurisdictional claims in published maps and institutional affiliations. 